\title{
ERJ
}

Engineering Research Journal

Faculty of Engineering

MenoufiaUniversity

\section{INCEPTION OF CAVITATION BUBBLES IN DIESEL ENGINES WET CYLINDER LINERS}

\author{
M. A. Hosien and S. M. Selim \\ Mechanical Power Engineering Department, Faculty of Engineering, Menoufyia \\ University, Shebin El-Kom, Egypt \\ Corresponding Author Email: mohamed_abdelaziz14@yahoo.com
}

\begin{abstract}
An experimental investigation was conducted using vibratory device in order to obtain the effects of the vibration frequency, static pressure, water temperature and depth of water between the liner and the water jacket on the initiation of cavitation bubbles. The initiation of cavitation bubbles was determined visually by naked eye with the help of stroboscopic lighting. The results indicated that the initiation of cavitation bubbles decreased with increasing vibration frequency and water temperature. In addition, cavitation bubbles initiation increased as the depth of water increased. The results also showed that the initiation of cavitation bubbles was independent of the static pressure. Results suggest that the designer should design the engine with cylinder liner vibration amplitude less than the critical vibration amplitude for initiation of cavitation bubbles to avoid the occurrence of cavitation. The results of cavitation bubbles initiation measurements were compared with a proposed cavitation inception model and good agreement was obtained.

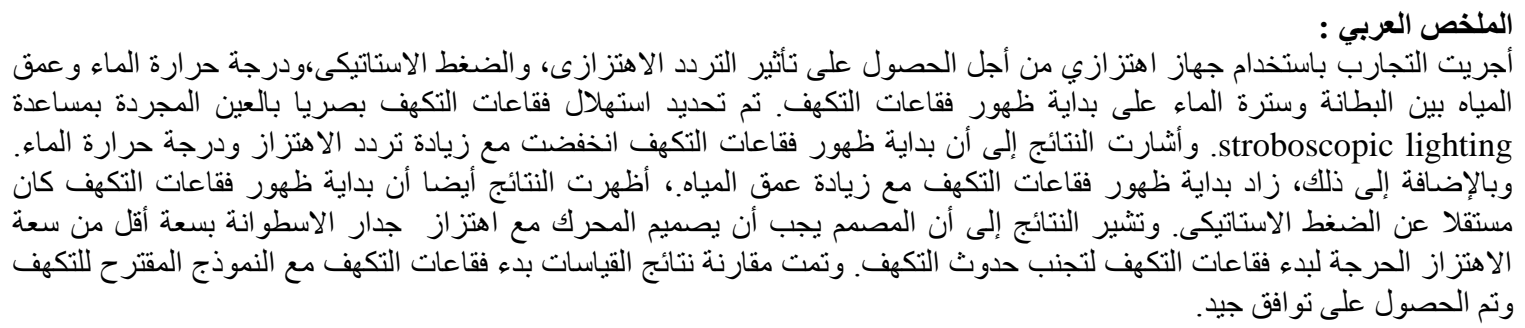

Keywords: Cavitation, inception, vibration, cylinder liner.

\section{INTRODUCTION}

Erosion is one of the most serious effects of cavitation on diesel engines wet cylinder liners and its significance has become more important with increasing engine speed and output. Cavitation normally occurs whenever the pressure at a point in a liquid is reduced below its vapour pressure at the corresponding liquid temperature. Cavitation erosion causes deep local holes on the cylinder liner surface [1]. Erosion of cylinder liners causes serious problems to reduce the reliability and life of the engine. Therefore, avoidance of cavitation is essential for the design of reliable diesel engine. Subsequently, reliable information on how cavitation bubbles initiation may vary with the design and operating parameters of wet cylinder liners is necessary to avoid the occurrence of cavitation. Although by now past researches [24] provide a reasonable guide to the relative erosion resistance of materials they don't enable the diesel engine designer to choose the practical design parameters at which engines should work to avoid cavitation.

Cavitation bubbles generally originate from microscopic bubble nuclei typically gaseous micro bubbles which are existed in the liquid [58]. The explosive growth of bubble nuclei in the liquid is usually defined by the term cavitation inception, i.e., cavitation inception is the limiting regime between the non-cavitating and the cavitating flow.

Inception of cavitation bubbles produced in cylinder cooling water is as a result of cylinder liners vibration. Cavitation can appears within the cooling water as a consequence of vibration of cylinder liners oscillating pressure fields are created over the free surface of the cooling liquid. Acoustic cavitation bubbles may appear within the liquid if the vibration amplitude is large enough to cause rupture in the liquid when the negative oscillation occurs. 


\section{A. Hosien and S. M. Selim "INCEPTION OF CAVITATION BUBBLES IN DIES..."}

Practically, inception of cavitation bubbles identified as either the visual appearance of cavitation bubbles or acoustical emission. Sound emission usually occurs earlier than the visual identification of cavitation inception because microscopic bubbles, invisible to naked eye emit pressure impulses before can see with further reduction of pressure. Many investigator performed experimental work using signals of noise and vibration which used for detection the onset of cavitation in various structures summaries of work can be found in [9-17]. Their results demonstrated that the successful use of the acoustic and vibration techniques for detecting incipient cavitation. Hosien and Selim [18] compered visual and acoustic methods for the detection of cavitation inception and concluded that at the onset of cavitation there was a marked rise of the sound pressure level in the high-frequency range.

Mahle Gmb [19] reported that two main factors control the cause of cavitation in cylinder liners. These factors are the flow conditions of the coolant and the vibrations of the cylinder. Kruger et al. [20] performed a numerical study to analyze the water flow inside MWM INTERNATIONAL's high-speed diesel engine. They stated that a modification on the geometry leads to an avoidance of the occurrence of cavitation at water jacket regions.

It is appeared from the brief review of the previous researches that the effects of various parameters such as vibration frequency of the cylinder liner, depth between liner and cooling water jacket, static pressure and temperature of engine cooling water on the initiation of cavitation bubbles have been given little concern. Obviously there is clearly a need for more data on the effects of various operating and design parameters upon the initiation of cavitation bubbles to help the high speed diesel engines designer to avoid the cavitation likely to occur in his engine. For this purpose the present work is devoted to study experimentally the effects of vibration frequency, depth between liner and cooling water jacket, static pressure and temperature of cooling water.

\section{SEMI-EMPIRICAL MODEL FOR VIBRATORY CAVITATION INCEPTION}

Cavitation inception is the beginning of cavitation. It is well known that cavitation inception occurs as a consequence of the rapid growth of small nuclei that have become unstable due to a change in ambient pressure.
These nuclei can be either presented in the flow or find their origins in small cracks or crevices at the boundary surface of the flow.

Most of the progress in understanding the details of the inception process has been made through the consideration of the dynamic equilibrium of a spherical bubble containing vapour and noncondensable gas. The Rayleigh- Plesset [21] equation describes this equilibrium:

$\mathrm{RR}^{\ddot{2}}+\frac{3}{2} \dot{\mathrm{R}^{2}}=1 / \rho\left[\mathrm{P}_{\mathrm{V}}+\mathrm{P}_{\mathrm{g}}-\mathrm{P}-\frac{2 \mathrm{~S}}{\mathrm{R}}-4 \mu \frac{\dot{\mathrm{R}}}{\mathrm{R}}\right]$

Where $R$ is the bubble radius, $P_{v}$ the vapour pressure, $\mathrm{P}_{\mathrm{g}}$ the partial pressure of noncondensable gases, $\mathrm{P}$ the external pressure, $\mathrm{S}$ the surface tension, $\mu$ the dynamic viscosity, and $\rho$ the liquid density. Dote denotes a differentiation with respect to time. It is generally agreed that dynamic effects can be important when the time available for growth i.e., exposure time is shorter than about 1.0 milliseconds. Dynamic effects are especially important in ultrasonic, where exposure time per cycle is less than 0.03 millisecond [21]. Therefore, the dynamical terms in Rayleighplesset equation may be neglected in the present study.

Hence, Rayleigh-Plesset equation (1) reduces to

$\mathrm{P}=\mathrm{P}_{\mathrm{V}}+\mathrm{P}_{\mathrm{g}}-\frac{2 \mathrm{~S}}{\mathrm{R}}$

Clearly, such a bubble may begin to grow if:

$P<\mathrm{P}_{\mathrm{V}}+\mathrm{P}_{\mathrm{g}}-\frac{2 \mathrm{~S}}{\mathrm{R}}$

Now, if it is assumed that the temperature and mass of the gas in the bubble remains constant as the external pressure is reduced, then the pressure, $\mathrm{P}_{\mathrm{g}}$ for a given mass of gas will vary inversely with the volume of gas bubble (i.e., free to expand isothermally P.V = C) that is ,

$\mathrm{P}_{\mathrm{g}} \propto \frac{1}{\mathrm{R}^{3}}$ Thus the gas pressure can be

represented as:

$\mathrm{P}_{\mathrm{g}}=\left(\mathrm{P}_{\mathrm{o}}-\mathrm{P}_{\mathrm{V}}+\frac{2 \mathrm{~S}}{R_{o}}\right) \frac{\mathrm{R}_{o}^{3}}{R^{3}}$

where the subscript "o" refers to the initial value of the appropriate quantity.

Substituting (2) into (3), an expression for the external pressure is obtained; thus corresponds to the static equilibrium of bubbles differing in radius,

$\mathrm{P}=\mathrm{P}_{\mathrm{V}}+\left(\mathrm{P}_{\mathrm{o}}-\mathrm{P}_{\mathrm{V}}+\frac{2 \mathrm{~S}}{R_{o}}\right) \frac{\mathrm{R}_{o}^{3}}{R^{3}}-\frac{2 \mathrm{~S}}{\mathrm{R}}$

A bubble, whose radius is $R_{0}$ at the initial ambient pressure $\mathrm{P}_{0}$, explodes at the critical ambient pressure $P_{c}$. The pressure $P_{c}$ can be obtained by differentiating equation (4) with respect to $\mathrm{R}$, and setting the derivative equal to zero and is expressed by the following equation. 
$\mathrm{P}_{\mathrm{c}}=\mathrm{P}_{\mathrm{V}}-\left[\frac{2 \sqrt{3}}{9}\left(\frac{2 S}{R_{o}}\right)^{3 / 2} /\left(\mathrm{P}_{\mathrm{o}}-\mathrm{P}_{\mathrm{V}}+\frac{2 \mathrm{~S}}{R_{o}}\right)^{1 / 2}\right]$ ( 5)

Equation (5) shows that a large tensile strength is required to explode a small bubble. The minimum radius of the nucleus can be estimated from the observed minimum pressure using equation (5).

The theoretical determination of the external pressure $(\mathrm{P})$ as a function of vibration amplitude and frequency is extremely complex. In principle, knowledge of the factors affecting the external pressure is necessary to determine the inception of cavitation bubbles.

This point was considered by Moustafa [22]. He conducted an experimental study using magnetostriction vibratory. His experimental results showed that the driving pressure is proportional to water depth $(\mathrm{t})$ to the power

$(-1 / 2)$, (i.e., $\left.P \propto t^{-0.5}\right)$, that is,

$\mathrm{P} \propto K_{1} \mathrm{t}^{-0.5}$

Where $K_{1}$ is the constant of proportionality in equation (6).

When a cylindrical piston vibrates with its circular face inside the liquid, the acoustic pressure amplitude at the radiating surface is approximately given by:

$$
\mathrm{P}=\rho \mathrm{CA} \omega
$$

Where $\mathrm{A}$ is the amplitude of the vibratory end, $\omega$ is the circular frequency $(\omega=2 \pi \mathrm{f}), \mathrm{f}$ is the vibration frequency, $\mathrm{C}$ is the sound speed in liquid, and $\rho$ is the density of liquid.

By combining (6) and (7) $\mathrm{P}_{\mathrm{c}}$ can be given as follows:

$\mathrm{P}_{\mathrm{c}}=K_{1} 2 \pi \rho C \cdot \mathrm{A}_{\mathrm{c}} \mathrm{f} \cdot \mathrm{t}^{-0.5}$

Where, $A_{c}$ is the cavitation inception vibration amplitude.

Substituting (8) into (5), a new scaling law for inception of vibratory cavitation is given by:

K. $A_{c} \cdot f \cdot t^{-0.5}=$

$\left\{\mathrm{P}_{\mathrm{V}}-\left[\frac{2 \sqrt{3}}{9}\left(\frac{2 S}{R_{O}}\right)^{3 / 2} /\left(\mathrm{P}_{\mathrm{o}}-\mathrm{P}_{\mathrm{V}}+\frac{2 \mathrm{~S}}{R_{O}}\right)^{1 / 2}\right]\right\}$

Where $\mathrm{K}$ is constant, $\left(\mathrm{K}=2 \pi \rho \mathrm{CK}_{1}\right) . \mathrm{K}_{1}$ is constant of the proportionality in equation (6). In equation (9) $\mathrm{K}$ and $\mathrm{R}_{\mathrm{o}}$ can be estimated from relevant test results. The units are $\mathrm{kg} / \mathrm{m}$ for $\mathrm{S}$, $\mathrm{kg} / \mathrm{m}^{2}$ abs. for $P_{v}$ and $P_{o}, m$ for $R_{o}, m m$ for $\mathrm{A}, \mathrm{Hz}$ for $\mathrm{f}$ and $\mathrm{cm}$ for $\mathrm{t}$.

Equation (9) gives the cavitation inception vibration amplitude $\left(\mathrm{A}_{\mathrm{c}}\right)$ as a function of vibration frequency, water depth between liner and water jacket surfaces vapour pressure, surface tension, initial pressure of the liquid, initial radius of bubble, and the constant $\mathrm{K}$ which includes liquid density and speed of sound in the liquid.
This equation is valid for vibratory cavitation. Equation (9) is used to predict the inception vibration amplitude of cavitation at a given operating condition.

\section{EXPERIMENTAL DETAILS}

\subsection{Experimental Equipment}

The equipment used in the present investigation is a vibratory device in which vibration frequency and amplitude can be varied independently. This vibratory device was designed simulates the vibrations of the cylinderliner.

Figure 1 shows the experimental arrangement.

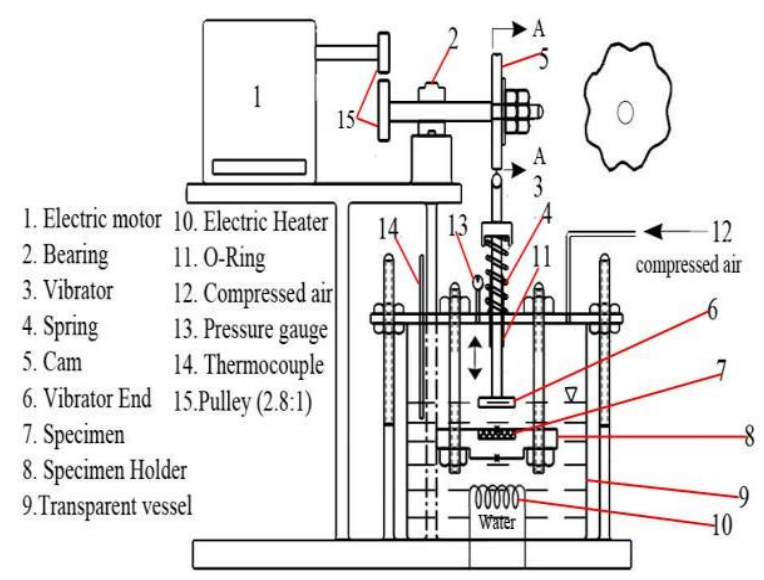

Fig.1. Diagrammatic arrangement of an experimental vibratory apparatus.

The vibrator was driven by a 1000 Watt variable speed universal motor. The face diameter of the vibrator end is $25 \mathrm{~mm}$. The amplitude of vibration could be varied from zero to $4 \mathrm{~mm}$. The maximum vibration frequency is of the order of $450 \mathrm{~Hz}$. The pressure wave generated resulted in the formation of cavitation cloud in the gap between the vibratory end and the face of the specimen. Specimen holder was fixed to the apparatus frame and positioned coaxial with the vibratory end. The vibratory end was mounted by an $\mathrm{O}$-ring arrangement into a sealing flange that the air space between water surface and flange can be pressurized as desired. An electric heater was mounted into the water container to maintain the water at the required temperature. Using closed container enables apparatus users to have much closer control of the temperature and pressure of water. The container was fabricated from transparent Perspex to permit visual observations. 


\subsection{Tests Performed and Procedures}

The tests were divided into constant temperature sets for varying depth, frequency, and amplitude, constant pressures sets for varying amplitude frequency with constant water depth and temperature. Six temperatures, ranging from 40 to $115{ }^{\circ} \mathrm{C}$, four pressures, ranging from 1 to $4 \mathrm{~atm}$. and four water depth values ranging from 2 to $8 \mathrm{~cm}$ were used. The expected errors in measuring frequency, amplitude, depth of water and temperature of water are $\pm 2 \%, \pm 3 \%, \pm 1.5 \%$ and $\pm 2 \%$ respectively.

The experimental tests were conducted to study the effects of various parameters on the initiation (inception) of cavitation bubbles.

Inception of cavitation refers to the first appearance of tinny cavitation bubbles zone. The vibration amplitude and frequency, water depth, physical properties and temperature of the liquid determined the conditions under which cavitation inception takes place.

The temperature of the water in the container was maintained at the desired temperature by the electric heater. The vibration frequency was adjusted manually until the required frequency was reached. Visual observations by naked eye with the help of stroboscopic light through the closed transparent container started from an arbitrary vibration amplitude.

The vibration amplitude then increased step by step until the inception of cavitation bubbles just become visible to the naked eye. The measurements of vibration amplitude and frequency, water temperature and depth corresponding to the inception of cavitation bubbles were recorded.

In order to obtain repeatable results on cavitation inception, the vibration amplitude was further increased beyond the inception until cloud of cavitation was observed during the negative pressure part of the sin wave.

Therefore, the vibration amplitude was decreased slowly until the cavitation bubble disappeared, i.e., desinent cavitation. Desinent cavitation means the disappearance of cavitation. The disappearance of the last small cavitation bubbles can usually be noted visually. The measurements of vibration amplitude and frequency and depth corresponding to the desinent conditions were recorded. These visual observations were repeated for many other frequencies, temperatures, water depths and pressures and the corresponding readings were determined.
Tap water from laboratory of Faculty of Engineering, Menoufia University was used.

Exploratory preliminary inception and desinent tests were performed. The results of these tests indicated that no significant differences were observed between inception and desinent.

\subsection{Pattern of Vibratory Cavitation}

Direct visual observations using stroboscopic light through the transparent vessel indicated that a mass of bubbles (i.e. cloud) appeared to radiate outwards. The cavitation bubbles believed to be produced radially are actually born with a vertically scattered distribution within a semispherical space. In the case of $2 \mathrm{~cm}$ water depth, cluster of bubbles appears on the center of the vessel bottom, which sometimes disappears again, but mostly stays during the whole period of existence of the cloud.

In the inception stage where the cavitation bubbles just become visible to the naked eye, a few scattered bubbles are seen around the central region of the vibratory surface.

\section{INCEPTION EXPERIMENTAL RESULTS AND DISCUSSION}

The visual observations of inception of cavitation bubbles were carried out for various test conditions and are plotted against vibration frequency in Figures 2 to 8.

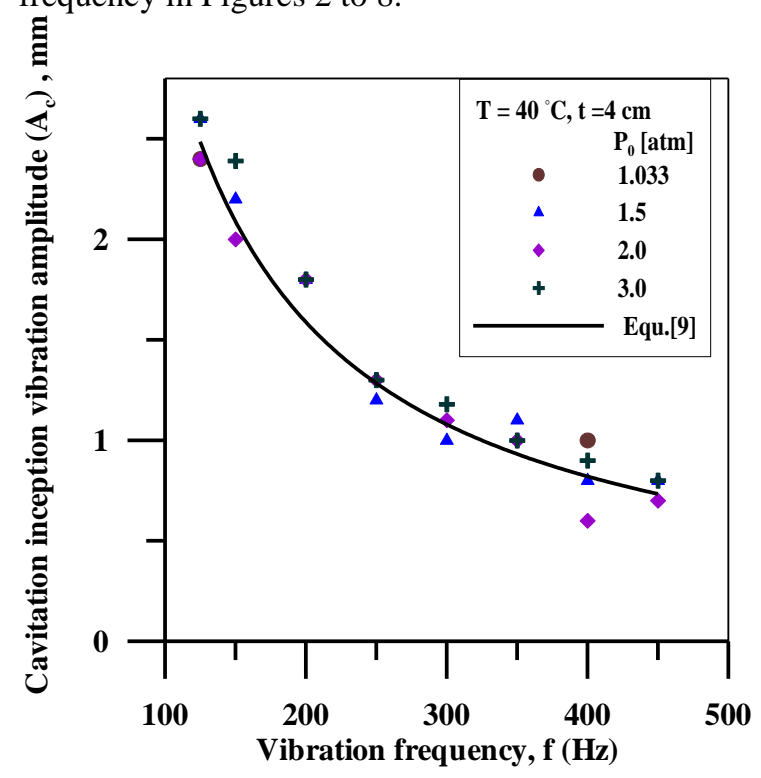

Fig.2. Variation of cavitation inception vibration amplitude with vibration frequency at various vessel pressures. 


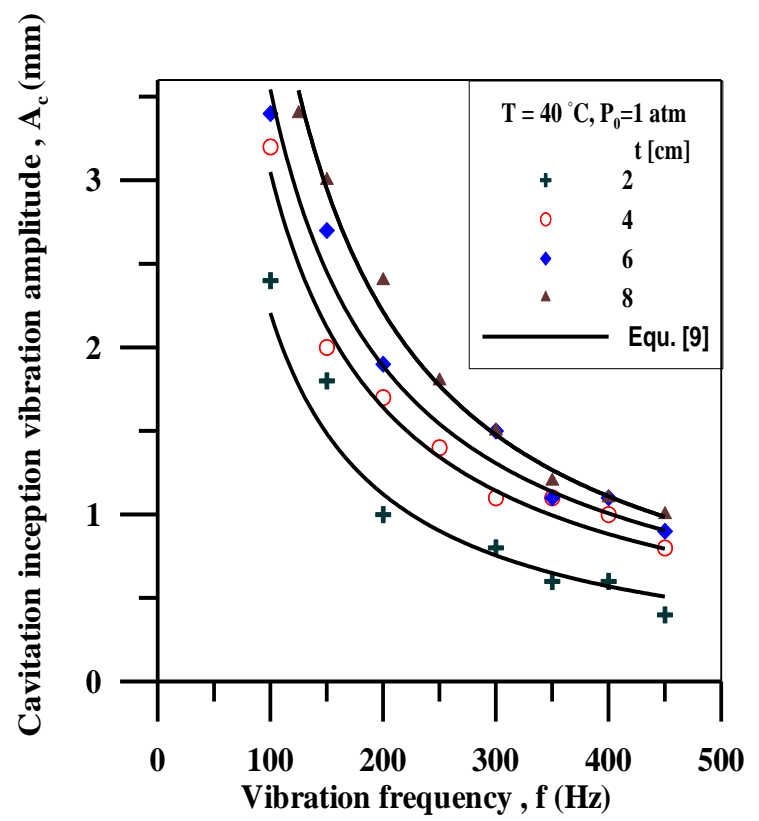

Fig.3. Variation of cavitation inception vibration amplitude with vibration frequency at various water depths ( $t)$.

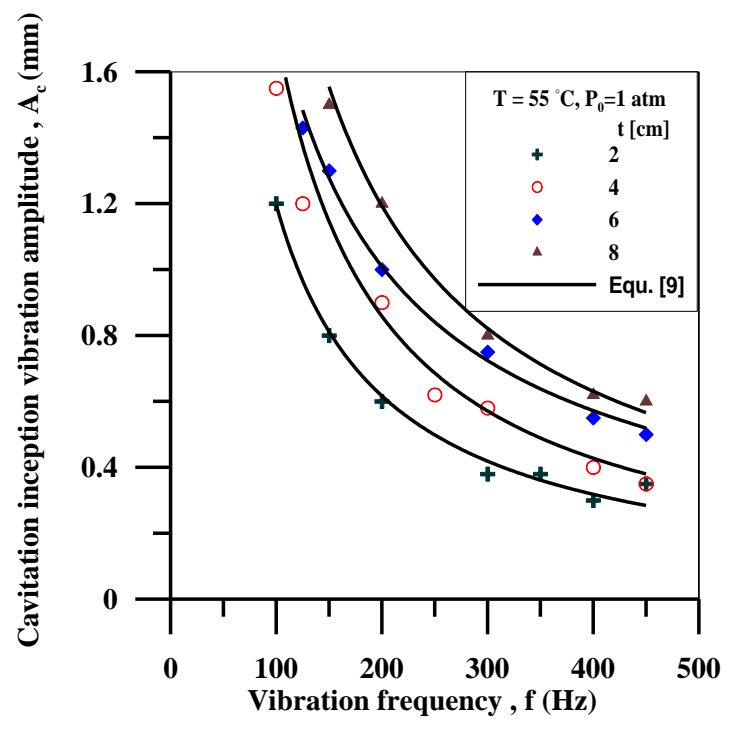

Fig.4. Variation of cavitation inception vibration amplitude with vibration frequency at various water depths $(t)$.

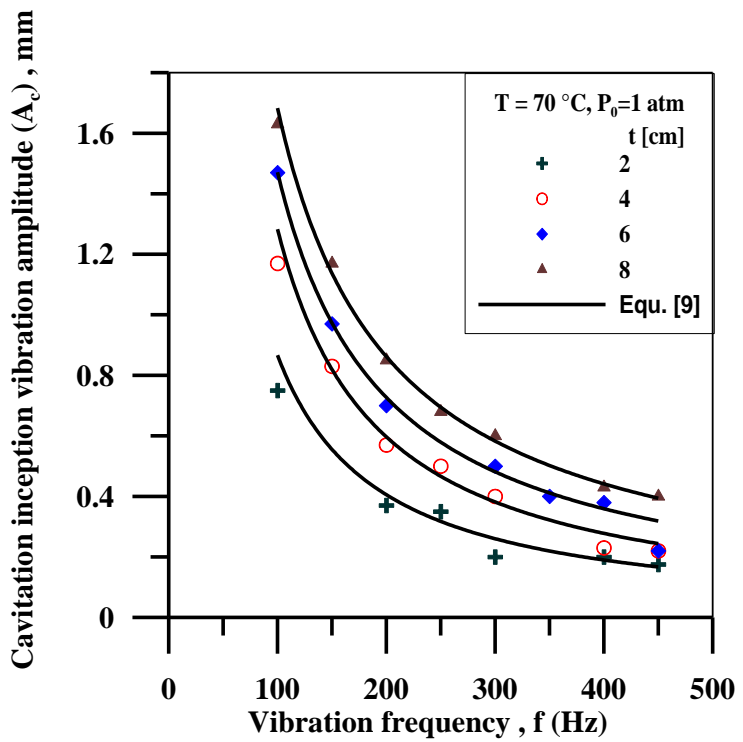

Fig.5. Variation of cavitation inception vibration amplitude with vibration frequency at various water depths $(t)$.

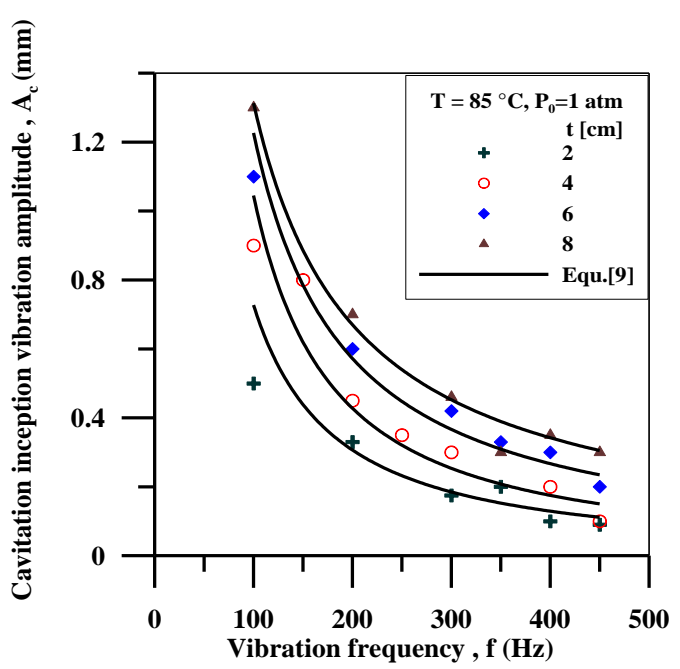

Fig.6. Variation of cavitation inception vibration amplitude with vibration frequency at various water depths $(t)$. 


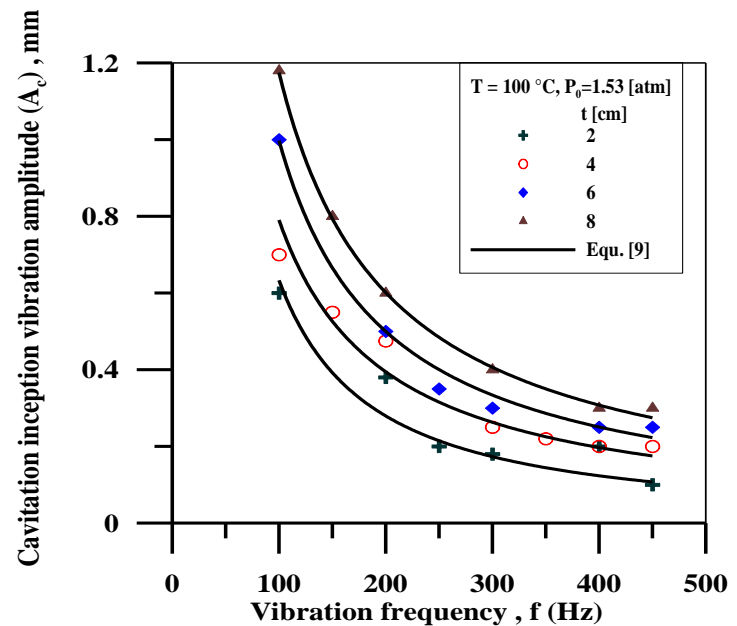

Fig.7. Variation of cavitation inception vibration amplitude with vibration frequency at various water depths $(t)$.

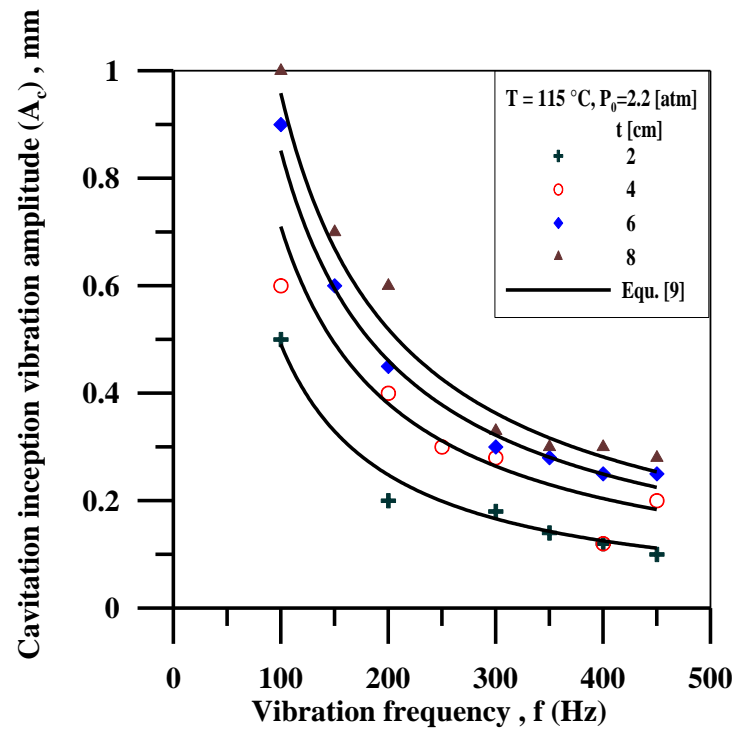

Fig.8. Variation of cavitation inception vibration amplitude with vibration frequency at various water depths $(t)$.
Figure 2 shows the effect of applied static pressure on the surface of water on the inception vibration amplitude at various vibration frequencies with a constant temperature of $40{ }^{\circ} \mathrm{C}$ and water depth of $4 \mathrm{~cm}$. This Figure shows that the inception vibration amplitude of cavitation is found to be strongly dependent on the vibration frequency and as the vibration frequency increases the vibration amplitude of cavitation inception decreases. It shows that for a given vibration frequency the inception vibration amplitude is independent of the static pressure acts on the surface of the water in the vessel. A possible explanation for this trend is that the tensile strength of the water corresponding to inception of cavitation is independent of the static pressure to which the water is subjected and surface tension forces play a great role on the tensile strength of water.

The variation of the cavitation inception amplitude with vibration frequency at fixed water depth is shown in Figures 3 to 8 for temperature values ranging from 40 to $115^{\circ} \mathrm{C}$. In general, these Figures show that the inception vibration amplitude of cavitation increases rapidly with decreasing the vibration frequency. This trend is reasonably confirmed by the acoustic theory which indicates that the acoustic pressure amplitude generated at the vibratory face is proportional to the vibration amplitude and the vibration frequency. Therefore, a certain level of vibration frequency and vibration amplitude is required to initiate cavitation. The effect of temperature on the inception vibration amplitude of cavitation at fixed frequencies and constant water depth of $2 \mathrm{~cm}$ shown in Figure 9 indicates quite clearly that the inception vibration amplitude of cavitation increases as the temperature of the water decreases. The consequences of increasing water temperature are to decrease the gas content, increase the initial size of nuclei, increase the vapour pressure and change the speed of sound in the water. The increase in vapour pressure and nuclei size with temperature and the gas content effects are all related to the decrease in the inception vibration amplitude with the increase in temperature at a given frequency and a given water depth. 


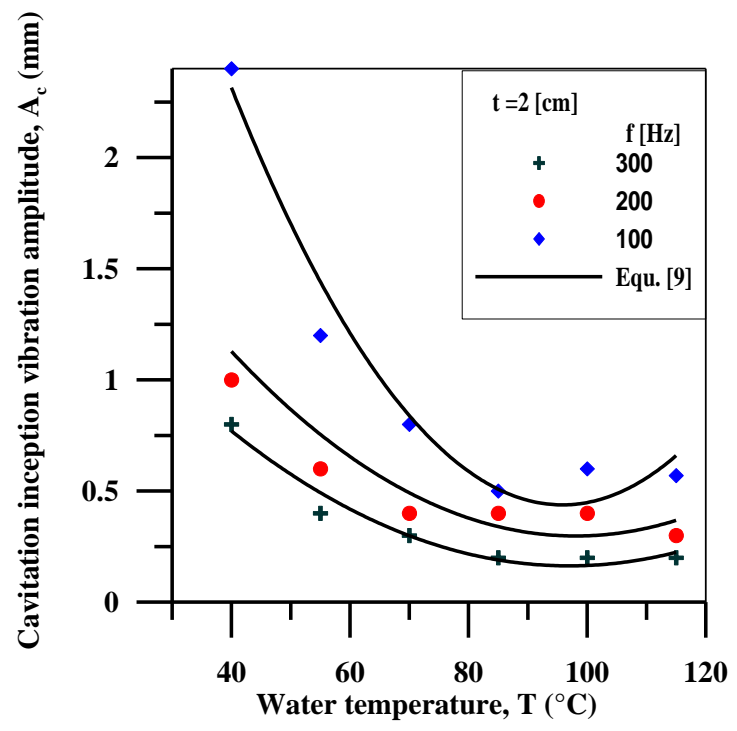

Fig.9. Variation of cavitation inception vibration amplitude with water temperature at fixed vibration frequencies.

Figure 10 indicates the variation of the cavitation inception vibration amplitude with water depth at fixed frequencies and a constant temperature of $55{ }^{\circ} \mathrm{C}$. This Figure shows that the inception vibration amplitude of cavitation increases as the water depth increases. The reason for this is that the amplitude of the threshold pressure to initiate cavitation is the sum of the amplitude of the acoustic pressure wave and the amplitude of the reflected pressure wave from the vessel base. The factors affecting the amplitude of the reflected pressure wave at the vessel base are the water depth, the vibration amplitude and frequency, the attenuation of the acoustic pressure wave and the physical properties of the water. Therefore, at a constant value of vibration frequency the water depth has an effect on the cavitation inception vibration amplitude as a result of the wave reflections in the water.

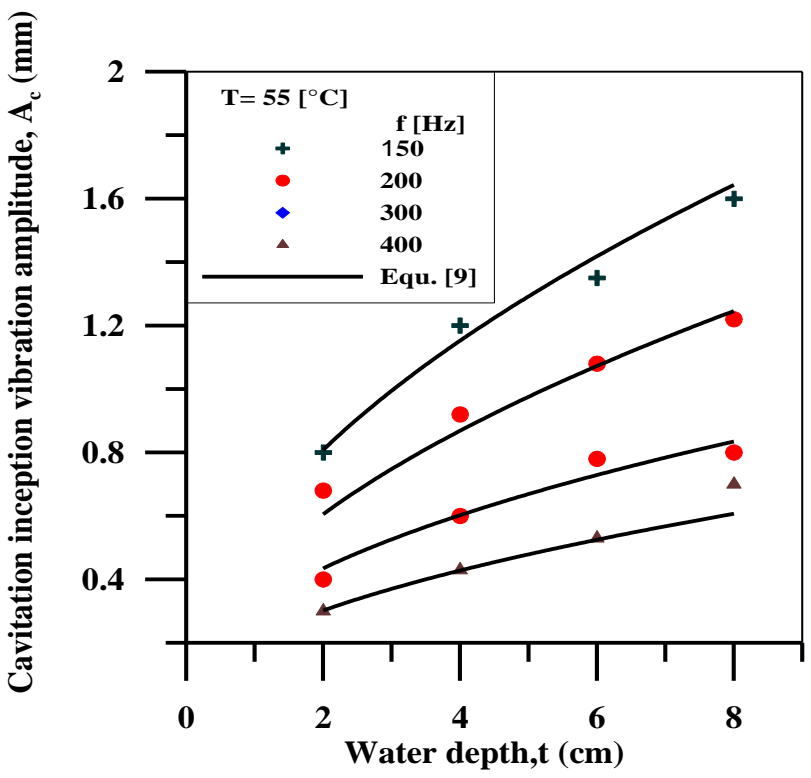

Fig.10. Variation of cavitation inception vibration amplitude with water depth at various frequencies.

\section{COMPARISON BETWEEN CAVITATION INCEPTION MODEL AND EXPERIMENTS}

Figures 2 to 10 show that the calculated cavitation inception amplitudes using equation (9) agree well with those measured for all the present data.

Figure 11 shows a good correlation, the correlation coefficient is in fact found between the measured and calculated cavitation inception amplitudes for all the present data. The correlation coefficient for liner regression is 0.98775. It is interesting to note that the calculated cavitation inception vibration amplitudes have been obtained using $\mathrm{R}_{\mathrm{o}}=50 \mu \mathrm{m}$ and $\mathrm{K}=4.05 \times 10^{-7} \mathrm{~T}^{4.4}$ in equation (9). The values of $\mathrm{K}$ and $\mathrm{R}_{\mathrm{o}}$ in equation (9) were computed using two cavitation inception variables amplitude $\left(\mathrm{A}_{\mathrm{c}}\right)$ from experimental results with constant water pressure, depth, and temperature at two different values of vibration frequencies. $K$ and $R_{0}$ were found to be independent of various test conditions. The agreement between the model and experiments means that the roles played by vibration amplitude, vibration frequency, water temperature and water depth are inconsistent with the present analyses. However, the analyses presented here are only a step towards better understanding the factors controlling the cavitation inception in vibratory system. 
These analyses showed that the phenomenon of cavitation inception is very complex and therefore several simplifying assumptions have been made according to the physics of the processes as currently understood. This is reflected in the derivation of relationship (9). Therefore, the present model represents an addition, to knowledge in this aspect which could help the diesel engine designer to avoid cavitation in his engine provided that the operating conditions are similar to that reported herein.

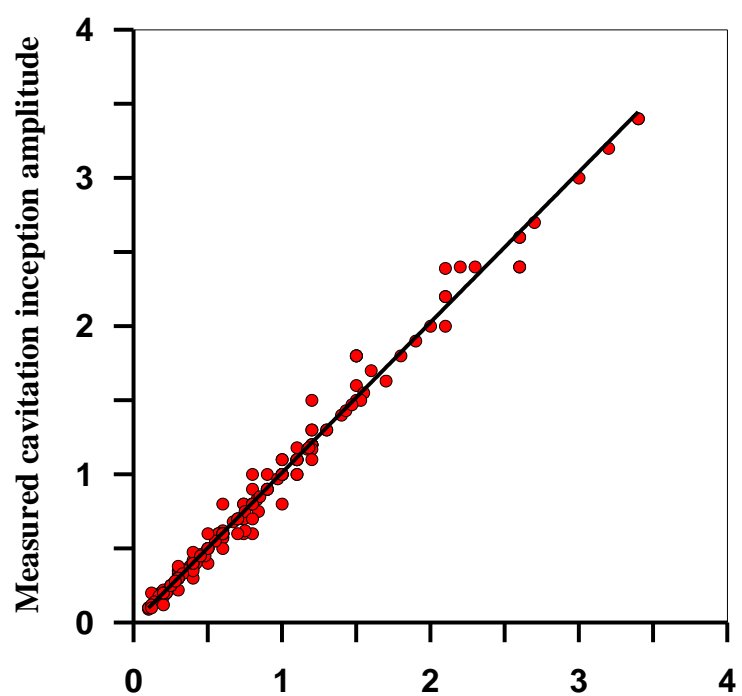

Calculated cavitation inception amplitude [ Eqn. 9]

\section{Fig.11. The measured cavitation inception amplitude versus the calculated cavitation inception amplitude using equation [9].}

\section{CNCLUSIONS}

The important conclusions that can be drawn are as follows:

1. The vibration amplitude at which the inception cavitation bubbles initiate decrease with the increasing vibration frequency and water temperature and decreased as the depth of water increased.

2. The predicted cavitation bubbles inception vibration amplitude from the proposed semi- empirical model was in good agreement with the present experimental results..

\section{REFERENCES}

[1] Yu-Kang, Z., Jiu-Gen, H. and Hammitt, F. G.," Cavitation erosion of diesel engine wet cylinder liners", Wear (1982), 76(3), 321-328.

[2] Franc, J. P., Riondet, M., Karimi, A. and Chahine, G. L., "Material and velocity effects on cavitation erosion pitting", Wear (2012), 274, 248-259.

[3] Choi, J. K., Jayaprakash, A. and Chahine, G. L., "Scaling of cavitation erosion progression with cavitation intensity and cavitation source", Wear (2012), 278, 5361.

[4] Hattori, S. and Kitagawa, T., "Analysis of cavitation erosion resistance of cast iron and nonferrous metals based on database and comparison with carbon steel data", Wear (2010), 269(5), 443-448.

[5] Washio, S., Kikui, S. and Takahashi, S., "Nucleation and subsequent cavitation in a hydraulic oil poppet valve", Proceedings of the institution of mechanical engineers, part C: Journal of mechanical engineering science (2010), 224(4), 947-958.

[6] Joseph, D. D., "Cavitation and the state of stress in a flowing liquid", Journal of Fluid Mechanics (1998), 366, 367-378.

[7] Franc, J.P. ,"Physics and control of cavitation", Educational Notes RTO-ENAVT-143(2006), Paper 2, pp. 2-1 - 2-36.

[8] Borkent, B. M., Arora, M., Ohl, C. D., De Jong, N., Versluis, M., Lohse, D. and Khoo, B. C., "The acceleration of solid particles subjected to cavitation nucleation", Journal of Fluid Mechanics (2008), 610, 157-182.

[9] Apfel, R. E., "Acoustic cavitation inception", Ultrasonics (1984), 22(4), 167173.

[10] Hsiao, C. T. and Chahine, G. L., "Scaling of tip vortex cavitation inception noise with a bubble dynamics model accounting for nuclei size distribution", Journal of fluids engineering (2005), 127(1), 55-65.

[11] Černetič, J. and Čudina, M., "Cavitation noise phenomena in centrifugal pumps", 5th Congress of Alps-Adria, Acoustics Association, 12-14 (September 2012), Petrčane, Croatia , NOI-01 Page $1-6$.

[12] Čudina, M., "Monitoring of cavitation by sound in audible range and some new proposals for prevention cavitation in kinetic pumps", 5th Congress of Alps-Adria Acoustics Association (2012) 12-14, Petrčane, Croatia. 


\section{A. Hosien and S. M. Selim "INCEPTION OF CAVITATION BUBBLES IN DIES..."}

[13] Cudina, M. and Prezelj, J., "Detection of cavitation in the operation of kinetic pumps: Use of discrete frequency tone in audible pump spectra using audible sound", Applied Acoustics (2009) 70(4), 540-564.

[14] Gupta, S., Chouksey, V. K. and Srivastava, M., "Online detection of cavitation phenomenon in a centrifugal pump using audible sound", ITSI Transactions on Electrical and Electronics Engineering (ITSI-TEEE) (2013), 1, 103-107.

[15] Chudina, M., "Noise as an indicator of cavitation in a centrifugal pump" ,Acoustical Physics (2003), 49(4), 463-474.

[16] Park, C., Seol, H., Kim, K. and Seong, W., "A study on propeller noise source localization in a cavitation tunnel", Ocean Engineering (2009), 36(9), 754-762.

[17] Al-Hashmi, S. A., "Statistical analysis of acoustic signal for cavitation detection", International Journal of Emerging Technology and Advanced Engineering (2013).
[18] Hosien, M. A. and Selim, S. M. , "Acoustic detection of cavitation inception", Journal of Applied Fluid Mechanics (2017), Vol. 10, No. 1, pp. 31-40.

[19] Mahle GmbH, "Pistons and engine testing", ATZ/MTZ- Fachbuch. Wiesbaden Germany: Springer Vieweg (2012).

[20] Kruger, M., Kessler, M. P., Ataídes, R., de la Rosa Siqueira, C. , dos Reis, M. V. F., Mendes, A. S. and Argachoy, C., "Numerical analysis of flow at water jacket of an internal combustion engine", (No. 2008-01-0393). SAE Technical Paper (2008).

[21] Plesset, M. S. and Prosperetti, A., "Bubble dynamics and cavitation", Annual review of fluid mechanics (1977), 9(1), 145-185.

[22] Moustafa, S.M., "The effect of vibration water gap and pressure wave reflecting surface on cavitation erosion of cylinder liners", Proceedings of $1^{\text {St }}$ conference on Applied Mech. Eng. Military Tech College Cairo (1984). 